\title{
Síndrome de burnout y estilos de vida en docentes de la facultad de salud de una universidad privada de Perú en tiempos de pandemia
}

\author{
Burnout syndrome and lifestyles in teachers of the health faculty of a private \\ university in Peru in times of pandemic
}

\author{
Merlinda Valverde Asencios ${ }^{1}$
}

\begin{abstract}
RESUMEN
Objetivo: Determinar la relación entre Síndrome de Burnout y estilos de vida en docentes de la facultad de salud de una universidad privada en tiempos de pandemia. Metodología: De enfoque cuantitativo, diseño no experimental, tipo correlacional y de corte transversal. La muestra fue de tipo no probabilístico por conveniencia obteniendo un total de 120 participantes. Los instrumentos utilizados para la recolección de datos fueron el MBI versión Ed creado por Maslach, Jackson y Schwab en 1986 adaptado por Fernández (2002) y el cuestionario de estilo de vida FANTÁSTICO creado por Douglas et al. (1984) adaptado por Villar et al. (2016). Resultados: Existe correlación inversa y estadísticamente significativa entre estilo de vida y síndrome Burnout demostrado por un $p<.001$ y un rho $=-.461$; en cuanto a los resultados descriptivos; el $69,6 \%$ de los docentes de la facultad de salud presenta un nivel medio de síndrome de Burnout, el $42 \%$ presenta un estilo de vida adecuado. Conclusión: Existe relación inversa entre las variables de estudio, lo que demuestra que un estilo de vida saludable puede funcionar como protector para la salud mental de los docentes.
\end{abstract}

Palabras clave: Síndrome de Burnout, estilo de vida, docente universitario, universidad.

\begin{abstract}
Objective: To determine the relationship between Burnout Syndrome and lifestyles in teachers of the health faculty of a private university in times of pandemic. Methodology: Quantitative approach, non-experimental design, correlational type and cross-sectional. The sample was non-probabilistic for convenience, obtaining a total of 120 participants. The instruments used for data collection were the MBI version Ed created by Maslach, Jackson and Schwab in 1986 adapted by Fernández (2002) and the FANTASTIC lifestyle questionnaire created by Douglas et al. (1984) adapted by Villar et al. (2016). Results: There is an inverse and statistically significant correlation between lifestyle and burnout syndrome demonstrated by a $p<.001$ and a rho $=-.461$; in terms of descriptive results; $69.6 \%$ of the health faculty teachers present a medium level of Burnout syndrome, $42 \%$ present an adequate lifestyle. Conclusion: There is an inverse relationship between the study variables, which shows that a healthy lifestyle can function as a protector for the mental health of teachers.
\end{abstract}

Keywords: Burnout syndrome, lifestyle, professor, university

${ }^{1}$ RESOCENTRO, Lima, Perú

Orcid ID: 0000-0002-2959-5234 


\section{INTRODUCCIÓN}

Hablar sobre el síndrome de Burnout se ha vuelto una prioridad en esta década y aún más en la situación mundial que vivimos actualmente a causa de la pandemia por COVID 19, aunque no hay un contacto físico directo entre los docentes y estudiantes; quienes se han visto obligados a estar siempre disponibles para sus estudiantes, ocasionando en el docente agotamiento físico y emocional. Según Delgado (2020) durante este tiempo los docentes deben ser flexibles y estar disponibles en línea siempre, aunque estar disponible durante todo el día es admirable, es necesario que las maestras y maestros tengan tiempo libre para cuidarse, porque estar siempre trabajando puede generar muchos problemas en la salud dentro de los cuales es síndrome de Burnout.

Para González et al. (2016) el síndrome de Burnout es la respuesta al estrés laboral crónico, una vivencia que involucra sentimientos y actitudes con repercusiones nocivas para la empresa y la persona. Es cierto que el trabajo es una actividad beneficiosa para la economía del trabajador y para su salud mental colectiva e individual, sin embargo el mundo pasa por cambios constantemente a consecuencia de la industrialización, automatización, globalización y otros fenómenos económicos, políticos, naturales y sociales generan en la población trabajadora daño biológico, social o psicológico, tales como frustración, insatisfacción y estrés en sus lugares de trabajo, esto se ve reflejado en las estadísticas de la Organización Mundial de la Salud (2020), quienes afirman que casi dos tercios de la población mundial sufren de estrés laboral.

Gutiérrez et al. (2020) refiere que el Síndrome de Burnout, se produce principalmente en organizaciones y empresas que brindan servicios especialmente los profesionales que tratan directamente con clientes o usuarios, como docentes, médicos, enfermeras, policías, entre otros. Es por lo que la docencia es una de las profesiones más afectadas por el síndrome de Burnout. Uno de las razones para este acontecimiento es debido a que los docentes universitarios son el centro de expectativas de los alumnos y padres, la sociedad espera de ellos un ejemplo de vida dentro y fuera de las instituciones educativas, provocando así estresores psicosociales fuera de las funciones en el contexto institucional (Bruch, Aerts,
Guimarães, y Gonçalves, 2016). De igual manera el entorno laboral y en las condiciones de trabajo son otra causa de Síndrome de Burnout, actualmente a nivel mundial atravesamos una pandemia que nos ha confinado a permanecer aislados, las instituciones educativas fueron obligadas a cerrar las aulas presenciales y abrir aulas virtuales lo cual ha significado un esfuerzo doble para el docente universitario ya que muchos de ellos no contaban con los conocimientos necesarios de los programas y aplicaciones requeridas y tuvieron que adaptarse al aprendizaje remoto de emergencia, este entre muchos otros problemas que surgieron, para Acuña (2020) todos estos acontecimientos ha incrementado el Síndrome de Burnout en el docente provocando agotamiento emocional en el trabajo, la adopción de actitudes negativas hacia los estudiantes y la institución; así como el descontento, la desmotivación y la insatisfacción personal.

Sin embargo, investigaciones como la de Bicalho, Carvalho, Andrade, y Guimarães (2019) han demostrado que un buen estilo de vida ayuda a no desarrollar Síndrome de Burnout en docentes.

En el Perú, Choy (2017) constató que el 30,2\% de los docentes de una universidad privada presentaron síntomas altos de Síndrome de Burnout y este está se relacionó con un bajo desempeño laboral. Por otro lado, Albarracin, Castro, y Quevedo (2019) demostró que el $73.9 \%$ de docente de pregrado posee un nivel promedio o alto de Burnout, lo cual muestra que los docentes presentan un estado de presión y cansancio emocional como respuesta a la exposición de continuos estresores que repercuten emocionalmente.

Sin embargo, Ptáček et al. (2019) en su estudio en docentes del nivel escolar en República Checa, encontró que un estilo de vida más saludable se asocia con tasas de agotamiento más bajas; y que los maestros que se toman el tiempo para intereses familiares y personales tienen menor riesgo de padecer agotamiento que los que no lo hacen, sin embargo, en el estudio de Cardoso, Ferreira, Ferreira, y Nunes (2016) se encontró que el $90 \%$ de los docentes de enfermería son insuficientemente activos.

De acuerdo a lo expuesto, la presente investigación tiene como objetivo general determinar la relación que existe entre estilos de 
vida y síndrome de Burnout en docentes de la facultad de salud de una universidad privada de Perú.

\section{METODOLOGÍA}

Esta investigación es de enfoque cuantitativo, de diseño no experimental, de corte transversal, y de tipo descriptivo - correlacional (Hernández, Fernández y Baptista, 2014).

\section{Participantes}

La población estuvo conformada por los 174 docentes de los Campus de la Facultad de Ciencias de la Salud de una universidad privada de Perú.

La muestra fue de tipo no probabilístico por conveniencia, empleándose criterios de inclusión y exclusión. Los criterios de inclusión fueron: docentes que pertenezcan a la Facultad de Salud de los tres campus a tiempo completo o parcial, docentes que dicten clases a los estudiantes de alguna de las cuatro escuelas profesionales docentes que acepten participar del estudio. Por otro lado, los criterios de exclusión fueron: docentes invitados, docentes que ejerzan sólo funciones administrativas, docente que no acepte participar del estudio. Obteniendo así una muestra de 120 docentes participantes de esta investigación.

\section{Instrumentos}

Para la recolección de datos sobre las variables de estudio se emplearon un inventario y un test:

El Maslach Burnout Inventory en su versión Educators Survey o conocido por sus siglas MBI versión Ed fue creado por Maslach, Jackson y Schwab en 1986, con el objetivo de medir el afecto del síndrome Burnot en los docentes, constituido por 22 ítems con escala de medición tipo Likert de siete puntos, que va desde 0 (nunca) hasta 6 (todos los días), distribuidos en 3 dimensiones: agotamiento o cansancio emocional, despersonalización y realización personal. En Perú fue adaptado por primera vez por Fernández (2002) donde obtuvo una confiabilidad alfa de Cronbach de 0.78 en la dimensión agotamiento emocional; 0.76 en despersonalización y 0.74 en realización personal, en cuánto a la validez del instrumento se realizó a través de juicio de expertos donde obtuvo un $\mathrm{V}$ de Aiken de 0.89 a 1 en sus 22 ítems.

El cuestionario de estilo de vida FANTÁSTICO fue creado por Douglas et al. (1984) en Canadá originalmente compuesto por 9 dimensiones, debido a sus siglas en inglés, y con 25 preguntas. En su traducción y adaptación a español por el Consejo Nacional para la Promoción de la Salud, VIDA CHILE (2006), fue conformado por 30 preguntas distribuidas en 10 dimensiones; a su vez esta adaptación considero tres opciones de respuesta con valor numérico de 0 a 2 en una escala tipo Likert, donde 0: casi nunca; 1 : A veces; 2: Siempre, al puntaje final se le multiplica por 2, obteniéndose un puntaje final de 0 a 120. En Perú fue adaptado por Villar et al. (2016) con un alfa de Cronbach: de 0,778 demostrando así que es un cuestionario aceptable. El acrónimo FANTÁSTICO representa las primeras letras de sus diez dominios:

F: Familia y amigos

A: Asociatividad. Actividad física.

$\mathrm{N}$ : Nutrición

T: Tabaco

A: Alcohol. Otras drogas.

S: Sueño. Estrés.

T: Trabajo. Tipo de personalidad.

I: Introspección

C: Control de salud. Conducta sexual.

O: Otras conductas: (como peatón, pasajero del transporte público, sigo las reglas; uso cinturón de seguridad y tengo claro el objetivo de mi vida).

\section{Análisis de datos}

Se comenzó con el ingreso, codificación y limpieza de datos en el estadístico SPSS versión 24. Para el análisis de datos descriptivos se utilizaron tablas de frecuencias. En cuanto a la estadística inferencial, en primer lugar, se realizó la prueba de normalidad de KolmogorovSmirnov; la cual es indicada para muestras superiores a 50 sujetos, la variable síndrome de Burnout mostró un p-valor de ,067 lo que indica que no hay normalidad en su distribución por lo que se utilizó la prueba no paramétrica Rho de Spearman debido a la naturaleza de las variables. 


\section{RESULTADOS}

En la tabla 1 se evidencia que el $69,6 \%$ de los docentes de la facultad de salud presenta un nivel medio de síndrome de Burnout, el $24,1 \%$ un nivel bajo, por otro lado, únicamente el $6,3 \%$ de los docentes presenta un nivel de Burnout alto.

Tabla 1

Síndrome Burnout en los docentes de la facultad de salud.

\begin{tabular}{lll} 
Síndrome de Burnout & $\mathrm{n}$ & $\%$ \\
Baja & 27 & 24,1 \\
Media & 78 & 69,6 \\
Alta & 7 & 6,3 \\
Total & 112 & 100,0 \\
\hline
\end{tabular}

En la tabla 2 se evidencia que del $100 \%$ de los docentes el 0,9\% presenta estilo de vida fantástico, por otro lado, el $42 \%$ siendo la mayoría de los docentes, presenta un estilo de vida adecuado.

Tabla 2

Estilos de vida de los docentes de la facultad de salud.

\begin{tabular}{lll} 
Estilos de vida & $\mathrm{n}$ & $\%$ \\
Algo bajo, podrías mejorar. & 27 & 24,1 \\
Adecuado, estas bien & 47 & 42,0 \\
Buen trabajo, estas en el camino correcto & 37 & 33,0 \\
Felicitaciones, tienes un estilo de vida. Fantástico. & 1 &, 9 \\
Total & 112 & 100,0 \\
\hline
\end{tabular}

En la tabla 3 se observa que existe correlación inversa y estadísticamente significativa entre estilos de vida y síndrome Burnout demostrado por un rho= -.461 y $p<.001$, lo que permite rechazar la Ho, quedando demostrado que existe relación entre las variables.

Tabla 3

Análisis de correlación entre estilos de vida y síndrome burnout de los docentes de la facultad de salud.

\begin{tabular}{|c|c|c|c|}
\hline & & & Síndrome Burnout \\
\hline \multirow[t]{3}{*}{ Rho de Spearman } & Estilos de vida & Coeficiente de correlación &,- 461 \\
\hline & & $p$ & $<.001$ \\
\hline & & $\mathrm{n}$ & 112 \\
\hline
\end{tabular}

\section{DISCUSIÓN}

El síndrome de Burnout es conocido y estudiado desde 1976, su nombre surge de las palabras inglesas burn; que significa quemado, y out; exterior. Se manifiesta debido al estrés crónico, este llega a tal punto que el trabajador deja de sentir satisfacción laboral, sin embargo, es incapaz de salir de esa situación o trabajo. Se ha encontrado que este síndrome se surge debido a la demanda de interacción del trabajador con los clientes o usuarios del servicio, destacando así a los trabajadores del área de salud y docencia en todos sus niveles (Arias, Huamani, y Ceballos, 2019).

Diversos estudios han intentado descubrir las posibles causas de este síndrome en los 
docentes y sus efectos negativos en su labor docente y su repercusión en los alumnos. Por su parte Boas y Soares (2020) postulan que entre las principales causas para el desenvolvimiento del síndrome de Burnout en docentes están la aparición de nuevas tecnologías en los centros de educación, la falta de estructura física adecuada, la falta de actividades recreativas, el desinterés de los alumnos, la insatisfacción profesional, el cansancio emocional, violencia en las escuelas, desvalorización profesional y salarial; el hecho del profesor tener que estudiar para poder enseñar, cumplir con plazos y metas, las continuas reuniones que demandan esfuerzo y tiempo del profesor.

Por otro lado, bajo la actual realidad que enfrenta el mundo, debido a la pandemia por COVID 19, la educación a distancia o virtual fue el único medio utilizado; esto conllevo a que los docentes trasladaran la metodología tradicional que utilizaban a una donde la tecnología ya no fue simplemente de apoyo o complementaria, muy por el contrario, es el principal canal de enseñanza. Esto representó un gran reto puesto que el cambio fue muy repentino y a diferencia de los alumnos muchos de los docentes no son nativos digitales (González-Calvo, 2020). Para Barrenechea (2020), este proceso de adaptación a un entorno virtual podría ocasionar síndrome de burnout en los docentes debido a que no todos tienen la misma capacidad para brindar un aprendizaje digital y esto puede ocasionar una autovaloración de incompetencia; por su parte Padilla (2020) refiere que en la docencia digital existen factores aún más exigentes que en la presencial, dentro de ellas el no tener un horario específico, el uso de maquinaria propia, el estar disponible las 24 horas para los alumnos por el temor de no haber logrado la comprensión del tema impartido, son estas entre otras razones por las cuales el autor postula vez que "Si antes de la pandemia ya existía un desgaste emocional por parte del profesor, que se puede esperar ahora que los maestros deben adaptarse a una situación completamente diferente".

La presente investigación demostró que el $69,6 \%$ de los docentes de la facultad Ciencias de la Salud presenta un nivel medio de síndrome de Burnout, el $24,1 \%$ un nivel bajo, por otro lado, únicamente el $6,3 \%$ de los docentes presenta un nivel de Burnout alto.

Resultados similares se encontraron en la investigación de Vinueza, Barreno y Flores
(2020) quienes encontraron que el $72,41 \%$ de docentes de un centro de estudios superior se encuentra en riesgo de manifestar el síndrome de Burnout, el $24,14 \%$ lo presentaba y únicamente el $3,45 \%$ ausentaba este síndrome. Por su parte Padilla (2020) encontró que los docentes de un colegio el $88,1 \%$ tiene un nivel medio, el $11.9 \%$ un nivel bajo de síndrome de Burnout y ningún docente presenta un nivel alto.

Sin embargo, resultados distintos fueron encontrados por Lopez y Zacarias (2020) donde el $44,5 \%$ de los docentes universitarios presenta un nivel bajo de síndrome de Burnout, el $35,2 \%$ un nivel medio y el $20,3 \%$ presenta un nivel alto de síndrome de Burnout.

Como se pudo observar en el presente estudio los docentes en su mayoría presentan niveles medio o están en riesgo de presentar el síndrome Burnout, esto podría llegar a ser un gran problema no solo para las instituciones donde laboran sino también para sus alumnos ya que cuando los niveles de Burnout son altos el docente manifiesta por un trato impersonal, cínico y frío a los alumnos y colegas, los educadores con síndrome de Burnout actúan de manera similar a un robot que salen de su hogar, llegan a su centro de trabajo, imparten la clase, revisan los trabajos, responden inquietudes, vuelven a su hogar, todo esto sin sentir satisfacción. Esta situación no favorece al rendimiento de los alumnos ya que es sabido que un docente motivado incrementa la probabilidad de un aula armoniosa, si los educandos sienten al docente cercano a ellos es más factible que el curso tenga un buen rendimiento (Torres, (2020); Brito, (2018)).

Si nos detenemos a analizar las dimensiones del síndrome de Burnout y sus efectos en los docentes encontramos que en la dimensión agotamiento emocional manifiestan que a partir del confinamiento y la educación virtual surgió la incertidumbre si los estudiantes están comprendiendo la materia, si están realizando de manera adecuada la sesión educativa, a esto se le suma la inseguridad de continuar laborando en la siguiente gestión. Por otro lado, las anteriores causales del agotamiento mental continúan; elaborar las clases, los materiales, buscar información necesaria, solucionar conflictos de aprendizaje. En su investigación Torres (2020) encontró que el 55\% de los maestros con agotamiento emocional identificaron que la falta de motivación para dar sus clases era el principal síntoma. 
La dimensión despersonalización hace referencia a "sentirse extraño, una especie de doble yo, donde por un lado la persona se ve dónde está, pero por otro lado se ve o percibe sensaciones como si se estuviera desdoblando" (Cano, 2019). Dentro de las manifestaciones de la despersonalización se encuentran la perdida de estabilidad emocional, el descontrol mental, y la falta de compromiso con los centros laborares, es decir, obedecer sin opinar o refutar, ya que está en un estado de depresión y estrés. Lopez y Zacarias, (2020) en su investigación postuló que los docentes que presentan despersonalización alta puede deberse a que no logran formar vínculos personales con sus alumnos a través de las pantallas.

La dimensión de realización personal es inversa, es decir cuando el docente presenta un nivel bajo de satisfacción personal manifiesta un nivel alto de Burnout, mayormente manifestado en el abandono de objetivos personales $y$ profesionales, la inmersión de una rutina sin satisfacción, abandono del vínculo familiar y sentimientos de soledad y frustración.

Con respecto a la segunda variable de estudio la presente investigación demostró que del 100\% de los docentes solamente el $0,9 \%$ presenta un estilo de vida fantástico, el $33 \%$ ha realizado un buen trabajo, por otro lado, el $42 \%$ siendo la mayoría de los docentes, presenta un estilo de vida adecuado y el $24,1 \%$ tiene un nivel bajo en la escala de estilo de vida FANTASTICO.

Sin embargo, Messier, Castro, y Céspedes (2016) demuestra que, los docentes de una universidad en Colombia, el 13,3\% presenta un nivel fantástico en su estilo de vida, el $50 \%$ realiza un buen trabajo, el $16,7 \%$ tiene un nivel adecuado y un $20 \%$ de los docentes presentaron niveles bajos en la escala de estilo de vida, lo que nos indica que existe en ellos riesgo de desarrollar diversas patologías.

Canqui, (2020) en su estudio encontró que el $69,1 \%$ de los participantes tienen un estilo de vida medio o regular, el $28,2 \%$ tiene un nivel alto o bueno y solamente el $2,7 \%$ presentó un nivel bajo de estilo de vida. Los datos encontrados en el presente estudio fueron recolectados en medio del distanciamiento social lo cual puede explicar el menor porcentaje de docente con un estilo de vida fantástico, según Malta et al. (2020) durante el tiempo de confinamiento se redujo la práctica de actividad física, la ingesta de alimentos ultra procesados, la cantidad de cigarrillos incrementaron, de igual manera aumentó el tiempo frente a los dispositivos electrónicos y el tiempo de recreación disminuyó.

En la presente investigación las variables de estudio, síndrome de Burnout y estilo de vida, demostraron tener correlación inversa y estadísticamente significativa con un $p<.001$ y un rho $=-.461$. Lo que nos indica que si se tiene un de estilo de vida bueno o alto se obtendrá un nivel de síndrome de Burnout bajo, demostrando así que el estilo de vida puede funcionar como protector para la salud mental de los docentes.

Similares resultados encontraron Bicalho, Carvalho, Andrade, y Guimarães (2019) en los docentes de colegios públicos de Brasil, donde se halló una correlación negativa entre el síndrome de Burnout y estilo de vida, los autores concluyeron que un estilo vida saludable puede actuar como un factor de prevención y control del síndrome de Burnout en los docentes.

Porotra parte, Vásquez(2020)en su investigación encontraron relación entre el estilo de vida y el síndrome de Burnout en el personal de salud encontró los mismos resultados, llegando a la conclusión que existe relación significante entre las variables, demostrado que los trabajadores que tienen un estilo de vida saludable son menos propensos a tener Síndrome de Burnout.

Asimismo, se demostró que las personas que duermen el tiempo adecuado y o realizan ejercicios físicos con frecuencia no presentan el síndrome de Burnout a comparación de quienes no practican esos hábitos (Fernández, Pérez, y Peralta, 2017). También se encontró que la satisfacción familiar tiene correlación negativa con el síndrome de Burnout, lo que nos indica que cuanto mayor sea la satisfacción familiar que una persona sienta menores será su nivel de síndrome de Burnout (Arias, Huamani, y Ceballos, 2019).

Se entiende por estilo de vida al conjunto de conductas y comportamientos saludables o no para salud de la persona, al practicar un estilo de vida saludable se obtienen una infinidad de beneficios para el bienestar total, no solamente físico, también mental, emocional, espiritual y social. Es por ello que es imperativo promover e incentivar un estilo de vida saludable en los docentes, por el bienestar de los mismos, los alumnos y para el funcionamiento adecuado de las instituciones educativas. 


\section{Declaración de financiamiento y de conflictos de interés:}

El estudio fue financiado por los autores, quienes declaran no tener conflictos de interés.

\section{Correspondencia}

Merlinda Valverde Asencios

Correo electrónico:

merlindavalverde@upeu.edu.pe

\section{REFERENCIAS BIBLIOGRÁFICAS}

Acuña, L. A. (2020). COVID-19, burnout y educación: el rostro que no se ve - Educación Futura. Educación Futura, pp. 1-3. Retrieved from http:// www.educacionfutura.org/covid-19-burnout-yeducacion-el-rostro-que-no-se-ve/

Albarracin, C., Castro, E., \& Quevedo, J. (2019). Síndrome de Burnout y su relación con la satisfacción laboral en docentes del área de Inglés de pregrado en una universidad privada de Lima 2019-I. Universidad Tecnológica del Perú. Retrieved from http://repositorio.utp.edu. pe/bitstream/UTP/2319/1/Carmen Albarracin Elizabeth Castro_Janeth Quevedo_Trabajo de Investigacion_Maestria_2019.pdf

Arias, W., Huamani, J., \& Ceballos, K. (2019). Síndrome de Burnout en profesores de escuela y universidad: un análisis psicométrico y comparativo en la ciudad de Arequipa. Propósitos y Representaciones, 7(3), 72 . https://doi. org/10.20511/pyr2019.v7n3.390

Barrenechea, M. (2020). La gran prueba de la educación virtual: ¿Cómo un docente puede entusiasmar a sus estudiantes? https://rpp. pe/peru/actualidad/coronavirus-en-peru-lagran-prueba-de- la-educacion-virtual-como-undocente-puede-entusiasmar-a-sus- estudiantesen-clases-virtuales-noticia-1259300

Bicalho, C., Carvalho, M., Andrade, N., \& Guimarães, J. (2019). O estilo de vida influencia nos índices de burnout em professores. Brazilian Journal of Development, 5(10), 19160-19169. https://doi. org/10.34117/bjdv5n10-148

Boas, V. B., \& Soares, P. (2020). SÍNDROME DE BURNOUT EM DOCENTES: REVISÃO INTEGRATIVA SOBRE AS CAUSAS. Cuidarte Enfermagem, 14(1), 95-100.

Brito, J. (2018). Calidad educativa en las instituciones de educación superior: evaluación del síndrome de burnout en los profesores.
Revista Iberoamericana para la Investigación y el Desarrollo Educativo, 8(16). doi: https://doi. org/10.23913/ride.v8i16.356

Bruch, L., Aerts, D., Guimarães, G., \& Gonçalves, S. (2016). A Sindrome de Burnout(SB) em docentes do ensino superior de instituições privadas de Santarém, PA. Tempus Actas de Saúde Coletiva, 10(3), 115. https://doi.org/10.18569/tempus. v10i3.1872

Canqui, B. (2020). Percepción de la calidad, estilos de vida y Síndrome de Burnout en estudiantes de programa de doctorado EPG - UNA - Puno. Universidad Nacional del Altiplano.

Cano, A. (2019). Cuando la despersonalización acompaña a la ansiedad. Infosalus. https://www. infosalus.com/salud-investigacion/noticia-puede-ansiedad-causar-sintomas-despersonalizacion-20191009081439.html

Cardoso, B., Ferreira, T., Ferreira, B., \& Nunes, C. (2016). ESTILO DE VIDAE NÍVEL DE ATIVIDADE FÍSICA EM DOCENTES UNIVERSITÁRIOS. Revista Unimontes Científica, 18(1), 15-23. Retrieved from https://www.periodicos.unimontes. br/index.php/unicientifica/article/view/1820

Choy, R. (2017). Burnout y Desempeño Laboral en Docentes Universitarios de una Carrera en una Universidad Privada de Lima Metropolitana. UniversidadPeruana Cayetano Heredia. Retrieved from http://repositorio.upch.edu.pe/bitstream/ handle/upch/1011/Burnout_ChoyVessoni_ Rosana.pdf?sequence $=3$ \&isAllowed $=y$

Delgado, P. (2020). Burnout, el efecto de la cuarentena. Retrieved November 10, 2020, from https://sarrauteducacion.com/2020/08/31/ burnout-el-efecto-de-la-cuarentena/

Douglas, W., Nielsen, E., \& Ciliska, D. (1984). Lifestyle Assessment: Testing the FANTASTIC Instrument. Canadian Family Physician, 30, 1863.

Fernández, M. (2002). Desgaste psíquico (burnout) en profesores de educación primaria de Lima metropolitana. Persona, 5(1), 27-66. https://doi. org/10.26439/persona2002.n005.842

Fernández, J. C., Pérez, J. M., \& Peralta, M. I. (2017). Influencia de factores sociodemográficos, laborales y de estilo de vida sobre los niveles de burnout en personal sanitario de cuidados paliativos. An. Sist. Sanit. Navar, 40(3), 421-431. https://doi.org/10.23938/ASSN.0114

González-Calvo, G. (2020). Vivo y enseñando, pero... ¿por cuánto tiempo? Cuando las circunstancias sociales y los tiempos de pandemia convierten la pasión por la enseñanza en duda y 
desánimo. Márgenes, 1(3), 110-132. https://doi. org/10.24310/mgnmar.v1i3.8714

González, G., Carrasquilla, D., Latorre, G., Torres, V., \& Villamil, K. (2016). Síndrome de Burnout en docentes universitarios. Revista Cubana de Enfermeria, 31(4). https://doi.org/10.33890/ innova.v1.n9.2016.55

Gutiérrez, G., Naranjo, E., \& Tenecota, G. (2020). Síndrome de Burnout en el personal docente de la Facultad Ciencias de la Salud de la Universidad Técnica de Ambato. Universidad Técnica De Ambato.

Hernández Sampieri, R., Fernández Collado, C., \& Baptista Lucio, P. (2014). Metodología de la investigación: Roberto Hernández Sampieri, Carlos Fernández Collado y Pilar Baptista Lucio (6a. ed.). México D.F.: McGraw-Hill.

Lopez, E., \& Zacarias, H. (2020). Síndrome de Burnout en docentes universitarios durante de clases virtuales. Desafios, 11(2), 136-177. https://doi. org/10.37711/desafios.2020.11.2.209

Malta, D., Szwarcwald, C., Barros, M., Gomes, C., Machado, Í., Souza Júnior, P., ... Gracie, R. (2020). A pandemia da COVID-19 e as mudanças no estilo de vida dos brasileiros adultos: um estudo transversal, 2020. Epidemiologia e Servicos de Saude : Revista Do Sistema Unico de Saude Do Brasil, 29(4), e2020407. https://doi. org/10.1590/S1679-49742020000400026

Messier, S. P., Castro, D., \& Céspedes, C. (2016). Estilo de vida en docentes y administrativos de la Universidad de Santander Cúcuta. Semana de Divulgación Científica, 39-43.

Padilla Cayllahua, A. (2020). Clima laboral y síndrome de burnout durante las clases virtuales en docentes de un colegio de Ventanilla - 2020.

Ptáček, R., Vnukova, M., Raboch, J., Smetackova, I., Sanders, E., Svandova, L., ... Stefano, G. B. (2019). Burnout Syndrome and Lifestyle Among Primary School Teachers: A Czech Representative Study. Medical Science Monitor : International Medical Journal of Experimental and Clinical Research, 25, 4974-4981. https://doi. org/10.12659/MSM.914205
Torres, C. (2020). Síndrome Burnout y docentes universitarios (Caso: docentes universitarios bolivianos de la Universidad Autónoma Gabriel René Moreno). Eduacación Superior, VII(No 2), 14-24. Retrieved from http:// www.scielo.org.bo/scielo.php?pid=S2518$82832020000200005 \&$ script=sci_arttext\&tlng=es

Vásquez, P. (2020). Estilos de vida y síndrome de Burnout en el personal del Hospital II - 2 Tarapoto 2017. Universidad Nacional de San Martín Tarapoto. Universidad Nacional de San Martín Tarapoto. Retrieved from http://repositorio.unsm. edu.pe/handle/11458/3737

Villar, M., Ballinas, Y., Gutierrez, C., \& AbguloBazan, Y. (2016). Análisis de la confiabilidad del test FANTÁSTICO para medir estilos de vida saludables en trabajadores evaluados por el programa "Reforma de vida" del Seguro Social de Salud (EsSalud)Análisis de la confiabilidad del test FANTÁSTICO para medir estilos de $v$. Revista Peruana de Medicina Integrativa, 1(2), 17-26. Retrieved from https://www.researchgate. net/publication/306031784_analisis_de_la_ confiabilidad_del_test_fantastico_para_medir_ estilos_de_vida_saludables_en_trabajadores_ evaluados_por_el_programa_reforma_de_vida_ del seguro social de salud essalud\%0ahttp:// files/393/30603

Vinueza, C. N., Barreno, E., \& Flores, C. (2020). BURNOUT EN docentes de un Instituto Tecnológico Agronómico. CienciAmérica, 9(4), 91. https://doi.org/10.33210/ca.v9i4.349

Recibido: 02/02/2021

Aceptado: 25/05/2021 